\title{
Serological markers of extracellular matrix remodeling predict \\ transplant-free survival in Primary Sclerosing Cholangitis patients
}

\section{Running title: EXTRACELLULAR MATRIX markers predict clinical outcome in PSC}

Mette J. Nielsen ${ }^{1 *}$, Douglas Thorburn², Diana J. Leeming ${ }^{1}$, Johannes R. Hov, ${ }^{3,4,5,6}$, Ståle Nygård ${ }^{7,8,9}$, Bjørn Moum $^{4,10}$, Francesca Saffioti ${ }^{2,11}$, Odd H. Gilja ${ }^{12,13}$, Kirsten M. Boberg ${ }^{3,4,5,6}$, Giuseppe Mazza², Helge Røsj $\varnothing^{9,14}$, Massimo Pinzani' ${ }^{2}$, Tom H. Karlsen ${ }^{3,4,5,6}$, Morten A. Karsdal ${ }^{1}$, Mette Vesterhus ${ }^{3,12}$

${ }^{1}$ Fibrosis Biology and Biomarkers, Nordic Bioscience, Herlev, Denmark

${ }^{2}$ UCL Institute for Liver and Digestive Health, Division of Medicine, University College London \& Royal Free Hospital, London, UK

${ }^{3}$ Norwegian PSC Research Center, Department of Transplantation Medicine, Division of Surgery, Inflammatory Diseases and Transplantation, Oslo University Hospital Rikshospitalet, Oslo, Norway

${ }^{4}$ Institute of Clinical Medicine, University of Oslo, Oslo, Norway

${ }^{5}$ Section of Gastroenterology, Department of Transplantation Medicine, Oslo University Hospital, Oslo, Norway

${ }^{6}$ Research Institute of Internal Medicine, Oslo University Hospital Rikshospitalet, Oslo, Norway

${ }^{7}$ Bioinformatics Core Facility, Institute for Medical Informatics, Oslo University Hospital, Oslo, Norway

${ }^{8}$ Institute for Experimental Medical Research, Oslo University Hospital and University of Oslo, Oslo, Norway

${ }^{9}$ Center for Heart Failure Research, University of Oslo, Oslo, Norway

${ }^{10}$ Division of Medicine, Department of Gastroenterology, Oslo University Hospital, Oslo, Norway 
${ }^{11}$ Department of Clinical and Experimental Medicine, Division of Clinical and Molecular Hepatology, University of Messina, Messina, Italy

${ }^{12}$ National Center for Ultrasound in Gastroenterology, Haukeland University Hospital, Bergen, Norway

${ }^{13}$ Department of Clinical Medicine, University of Bergen, Norway

${ }^{14}$ Division of Medicine, Akershus University Hospital, Lørenskog, Norway 


\section{Footnote page}

*Corresponding author: Mette Vesterhus, Dept. of Internal Medicine, Haraldsplass Deaconess Hospital, P.box.6165, N-5892 Bergen, Norway; Phone: +47-55978500; email: vmet@ihelse.net

\section{LIST OF ABBREVIATIONS (in order of appearance)}

PSC: Primary sclerosing cholangitis; ELF: Enhanced liver fibrosis; PIIINP: N-terminal procollagen type III; HYA: Hyaluronic acid; TIMP-1: Tissue inhibitor of metalloproteinase; ECM: Extracellular matrix; MMP: Matrix metalloproteinase; UC: Ulcerative colitis; INR: international normalized ratio; AST: Aspartate aminotransferase; ALT: Alanine aminotransferase; ALP: Alkaline phosphatase; GGT: Gamma glutamyltransferase; PRO-C3: Marker of type III collagen formation; PRO-C5; Marker of type V collagen formation; C3M: Marker of type III collagen degradation; C4M: Marker of type IV collagen degradation; AUROC: Area under the receiver operator characteristics curve; OR: Odds ratio; HR: Hazard ratio; $\mathrm{Cl}$ : Confidence interval.

\section{Funding source:}

The study was sponsored by the Danish Science Foundation.

\section{Conflict of interests:}

MJN, DJL, and MAK are full-time employees at Nordic Bioscience. MAK holds stocks in Nordic Bioscience. MJN, DJL and MAK are among the original inventors and patent holders for PRO-C3, PRO-C5, C3M, and C4M. MV is an advisory board member for Intercept. 


\section{Authorship Statement}

$\mathrm{MV}$ is the guarantor of the article and supervised the project. MV, MAK, THK and MP conceived and designed the study. KMB, BM and JRH collected the biological samples and clinical data. DJL and MAK contributed to the designing of the laboratory analyses. MJN performed the laboratory analyses. DT, FS and GM contributed to the ELF Test analyses. MJN, SN and MV performed statistical analyses. HR and MV contributed to the designing and interpretation of statistical analyses. MJN, DT, JRH, OHG, KMB, MP, MAK, THK and MV contributed to the interpretation of the data. MJN and MV drafted the manuscript.

All authors reviewed the manuscript for critical content and approved the final version of the manuscript.

\section{Acknowledgements:}

The authors thank Liv Wenche Thorbjørnsen for assistance in the collection of serum samples. M. Pinzani and D. Thorburn gratefully receive funding from UCL NIHR BRC (funding 2017-2022) and PSC partners (funding 2017-2019).

Word count: 4466 (excluding Abstract and References) 


\section{ABSTRACT}

Background: Primary sclerosing cholangitis (PSC) is a progressive liver disease with a remarkably variable course. Biomarkers of disease activity or prognostic models predicting outcome at an individual level are currently not established. Aim: We aimed to evaluate the prognostic utility of four biomarkers of basement membrane and interstitial extracellular matrix remodeling in patients with primary sclerosing cholangitis. Methods: Serum samples were available from 138 large-duct primary sclerosing cholangitis patients (of which 102 [74\%] with inflammatory bowel disease) recruited 2008-2012 and 52 ulcerative colitis patients (controls). The median follow-up time was 2.2 (range 0-4.3) years. Specific biomarkers of type III and V collagen formation (PRO-C3 and PRO-C5, respectively) and type III and IV collagen degradation (C3M and C4M, respectively) were assessed. The Enhanced Liver Fibrosis (ELF) Test, including PIIINP, TIMP-1 and hyaluronic acid was assessed for comparison.

Results: All markers were elevated in primary sclerosing cholangitis compared to ulcerative colitis patients $(p<0.001)$. PRO-C3 showed the largest difference between the two groups with a 3-fold increase in primary sclerosing cholangitis compared to ulcerative colitis patients. Patients with high baseline serum levels of all markers, except C3M, had shorter survival compared to patients with low baseline serum levels $(p<0.001)$. Combining PRO-C3 and PRO-C5 the odds ratio for predicting transplant-free survival was 47 compared to the Enhanced Liver Fibrosis Test's odds ratio of 11.

Conclusions: Extracellular matrix remodeling is elevated in primary sclerosing cholangitis patients compared to ulcerative colitis patients. Furthermore, the interstitial matrix marker PRO-C3 was identified as a potent prognostic marker and an independent predictor of transplant-free survival in primary sclerosing cholangitis.

\section{Key words:}

Primary sclerosing cholangitis, liver fibrosis, cholestatic liver disease, liver, outcomes research 


\section{INTRODUCTION}

Primary sclerosing cholangitis (PSC) is a liver disease affecting the intra- and extrahepatic bile ducts. As the disease progresses, chronic inflammation leads to biliary fibrosis and ultimately end-stage liver disease. At present there is no effective medical treatment for PSC, while liver transplantation is the only option to improve patient survival in selected patients although the disease may recur. $(1,2)$ However, there is huge variation in the course of the disease with transplant-free survival ranging 12-21 years in different cohorts $(3,4)$ and with even larger inter-individual variation. There is an unmet need for early, accurate, noninvasive biomarkers to stratify risk of progression and adverse outcomes in PSC at the individual level. Currently, there are no established biomarkers for predicting disease progression or clinical outcomes of PSC (5). However, recent findings indicate that serological markers of fibrosis are promising prognosticators. The Enhanced Liver Fibrosis (ELF) test is a non-invasive, serum-based fibrosis marker panel based on three fibrosis markers which are all expressed during early stages of collagen deposition in early stages of fibrosis, namely the N-terminal procollagen type III (PIIINP), hyaluronic acid (HYA), and tissue-inhibitor of matrix metalloproteinase-1 (TIMP1). The ELF Test was demonstrated to be a potent prognostic marker in PSC for the prediction of transplant-free survival (6) and the findings were validated in an international multicenter setting (7).

In PSC, fibrosis develops with the typical portal to portal biliary fibrosis pattern and is in addition characterized by concentric periductal fibrosis with so-called onion-skin appearance (8). One possible method to differentiate early fibrosis from longer established PSC fibrosis is to study the extracellular matrix (ECM) remodeling in PSC. The extracellular matrix can be subdivided in two compartments; the basement membrane, which is the outer extracellular matrix on which epithelial and endothelial cells attach, and the interstitial matrix, which is the inner extracellular matrix (9). The basement membrane consists primarily of type IV collagen and laminin, whereas the interstitial matrix consists of fibrillar collagens type I, III, and V as well as smaller amount of other non-fibrillar collagens. Matrix 
metalloproteinases (MMPs) and their inhibitors (TIMPs) also play a pivotal role in extracellular matrix remodeling, favoring the tissue balance towards either more fibrogenesis or more fibrolysis (10).

The end product of tissue remodeling, i.e. a neo-epitope resulting from a specific protein cleaved by a specific protease, is released into circulation and may serve as biomarker for that pathological process. A range of serological biomarkers specifically targeting the extracellular matrix remodeling have been developed in recent years, many of which have been shown to be related to various types of liver diseases, as either diagnostic (11-13), prognostic $(14,15)$, or surrogate efficacy markers (16-18). By combining both the protease and the protein, these biomarkers reflecting the balance between fibrogenesis and fibrinolysis may better assess the dynamic activity of a disease state as opposed to a static representation of stage, compared to other biomarkers targeting the intact protein.

In this study we investigated four different extracellular matrix remodeling biomarkers reflecting both formation and degradation of the interstitial and basement membrane matrix, as prognostic markers for predicting transplant-free survival in PSC patients. 


\section{PATIENTS AND METHODS}

\section{Patient population}

The study population included 138 PSC patients, previously described (6). Serum samples were collected in the NoPSC Biobank from 2008-2012 with median follow up (range) 2.2 years (0.0-4.3) from serum sampling. Characteristics of the study population are shown in Table 1. Diagnosis of PSC was based on typical cholangiographic findings according to acknowledged criteria $(19,20)$. The first pathological cholangiography defined the time of diagnosis of PSC. Duration of PSC was defined as the time from the date of diagnosis to the date of serum sampling. Fifteen cases of PSC with features of autoimmune hepatitis were included. Cases of secondary cholangitis or small duct PSC were excluded. Control non-PSC sera from 52 ulcerative colitis (UC) cases ( 23 with active disease, 29 in remission) from a population-based Norwegian cohort were retrieved for comparison (21). All patients provided informed consent in writing. The protocol was in accordance with the Declaration of Helsinki and approved by the regional committee for research ethics in southeastern Norway (reference 2011/2572).

For PSC patients, patient records and the research database were revised for information on clinical and laboratory data, including ascites, encephalopathy, esophageal varices, variceal bleeding, inflammatory bowel disease status, colorectal or hepatobiliary malignancy, and medication at the time of serum extraction. Inflammatory bowel disease diagnosis was based on findings at colonoscopy and histology. Diagnosis of ulcerative colitis and Crohn's disease were established by accepted criteria. Updated information on liver transplantation dates and indications by December 31, 2012, were retrieved from the Norwegian/Nordic Liver Transplant Registry; and data on all-cause death by the same date were retrieved from the Norwegian Death Registry. No donor organs were obtained from executed prisoners or other institutionalized persons.

Biochemical analyses were performed using standard routine laboratory protocols for tests including platelets, creatinine, international normalized ratio (INR), aspartate aminotransferase (AST), alanine 
aminotransferase (ALT), alkaline phosphatase (ALP), and gamma-glutamyltransferase (GGT). The APRI (AST to platelet index) score was calculated using the published algorithm (22).

\section{Biomarkers of extracellular matrix remodeling}

We used validated competitive ELISAs (Nordic Bioscience, Herlev, Denmark) to assess true formation of interstitial matrix collagen type III and V (PRO-C3 and PRO-C5) and degradation of interstitial matrix collagen type III and basement membrane type IV collagen (C3M and C4M) in serum samples from PSC and ulcerative colitis patients. All biomarkers were assessed in a blinded manner according to the manufacturer (23-26) and samples were measured within the detection range.

\section{ELF Test and ELF score}

We analyzed frozen serum samples from the PSC patients using the commercially available ELF Test (Siemens Medical Solution Diagnostics, Inc., Tarrytown, NY). The assays for analysis of serum levels of TIMP-1, hyaluronic acid and intact N-terminal PIIINP were performed using the Siemens ELF Test kits containing assays designed specifically for the purpose of generating the ELF Test and an ADVIA Centaur XP analyzer (Siemens Medical Solutions Diagnostics, Inc.). In addition, an ELF score was calculated for PSC and ulcerative colitis patients and used for comparisons between these groups. For ELF score, commercial kits were used to analyze serum levels of TIMP-1 and, hyaluronic acid (both R\&D Systems), and intact Nterminal PIIINP was analyzed by radioimmunoassay (UniQ PIIINP RIA; Orion Diagnostica, Espoo, Finland) in frozen serum samples. The ELF score was then calculated using the published algorithm used in the ADVIA Centaur XP ELF Test, combining TIMP-1, HA, and PIIINP values: ELF score $=2.278+0.851 \ln \left(\mathrm{C}_{\mathrm{HA}}\right)+0.751$ $\ln \left(C_{P I I I N P}\right)+0.934 \ln \left(C_{T I M P-1}\right)(4)$. 


\section{Statistical analyses}

We tested continuous variables for normal distribution, and applied the Student $\mathrm{t}$ test or the MannWhitney $\mathrm{U}$ test as appropriate. Percentage differences for the levels of extracellular matrix remodeling markers between PSC and ulcerative colitis patients were calculated as the difference between PSC and UC divided by UC $\times 100 \%$. Data are presented as median (range). A primary endpoint was defined as all-cause death or liver transplantation, and a secondary endpoint was defined as all-cause death or liver transplantation due to hepatic decompensation. We generated Kaplan-Meier plots with patients subdivided according to tertiles and compared crude risk by the log-rank test. The discriminatory power of the ELF Test and extracellular matrix remodeling biomarkers was evaluated by the area under the receiver operating characteristics curve (AUROC) analysis, and differences between the AUROCs were compared with the method of DeLong (27). The optimal cut-off values to discriminate between patients with or without endpoints were obtained from the AUROC analysis according to Youden index. We censored data at 4 years for the Kaplan Meier analyses due to the low number of patients with longer follow-up. We explored the associations between baseline clinical and laboratory variables (APRI, ELF Test, PRO-C3, PROC5, C3M and C4M; and standard laboratory tests) and clinical outcome by univariate Cox proportional hazards regression analysis. Factors that were significantly associated with the outcome in the univariate analyses were entered into the multivariate model. Due to collinearity (rho>0.5) between PRO-C5, C3M and C4M on one hand, and PRO-C3, ELF Test and Mayo score on the other, we investigated the novel markers first in separate analyses and then collectively in an "all-in" model. The novel extracellular matrix markers were not normally distributed and therefore normalized to tertiles prior to Cox analyses, whereas nonnormally distributed standard laboratory tests (thrombocytes) were transformed by the natural logarithm. Odds ratios (OR) for predicting transplant free survival was calculated by $2 \times 2$ table comparing the lower and upper tertiles of each parameter. Hazard ratios (HR), odds ratios, and AUROCs are presented with 95\% confidence interval $(\mathrm{Cl})$. P values $<0.05$ were considered significant. Statistical analyses were performed 
using MedCalc (MedCalc Software, Ostend, Belgium) and SPSS (version 24, SPSS Inc., Chicago, IL, USA). Graphs were designed using GraphPad Prism version 7 (GraphPad Software, La Jolla, CA, USA).

\section{RESULTS}

\section{Patients}

Patient characteristics are shown in Table 1. The majority of patients were male with a median age at diagnosis of 34 years. Overall seventy four percent of PSC patients had inflammatory bowel disease (IBD) with ulcerative colitis affecting 59\%. Patients were followed for a median time period of 25.9 months, during which 33 patients underwent liver transplantation and 16 patients died. The indications for liver transplantation were decompensated liver disease $(n=9 ; 27 \%)$, intractable symptoms $(n=14 ; 42 \%)$ and biliary dysplasia ( $n=10 ; 30 \%)$. The median (range) Mayo risk score at serum withdrawal was 0.12 (-2.374.13). The control cohort consisted of 52 patients with ulcerative colitis without PSC, 23 of whom had active disease, whereas 29 were in remission.

\section{ELF Score compared to biomarkers of extracellular matrix remodeling in PSC and ulcerative colitis}

\section{patients}

All markers of extracellular matrix remodeling were significantly elevated in PSC patients compared to ulcerative colitis patients ( $p<0.001)$ (Figure 1). No differences between ulcerative colitis patients with active disease compared to patients in remission were observed for PRO-C3, PRO-C5, C3M or C4M (data not shown). Moreover, the type III collagen balance showed relatively more type III collagen formation (PROC3) compared to type III collagen degradation (C3M) in PSC patients as demonstrated by a higher percentage difference between PSC and ulcerative colitis patients for PRO-C3 compared to C3M (65\% vs. $14 \%)$. 
Similar to the extracellular matrix remodeling markers, the ELF score (Figure 1) and its individual markers, PIIINP, TIMP-1, and hyaluronic acid (Supplementary Figure 1), were also significantly elevated in PSC patients as compared to ulcerative colitis patients $(p<0.01-p<0.0001)$. No differences were observed between ulcerative colitis patients with active disease compared to patients in remission for neither ELF score nor its individual markers (data not shown).

\section{Performance of the ELF Test compared to biomarkers of extracellular matrix remodeling to discriminate between PSC patients with or without an end-point.}

In order to investigate prognosis, a primary end-point was defined as liver transplantation or death. In this study 47 of the PSC patients either underwent liver transplantation or died within the follow-up period, whereas 91 patients remained alive and transplant-free. AUROC analyses revealed that all four extracellular matrix remodeling biomarkers as well as APRI score and the ELF Test were able to discriminate patients who reached an endpoint from those who did not with similar AUROCs (Table 2), with a non-significant trend towards higher AUROC for Pro-C3 and Pro-C5 combined (AUROC 0.822).

Based on the cut-off values determined by the Youden index from the AUROC analyses for each novel marker and ELF Test, we separated the patients into a high risk group and a low risk group according to survival. All markers except C3M showed significantly shorter survival time in patients with high baseline marker levels compared to patients with low baseline marker levels (Table 3).

We then investigated the prognostic power of each of the markers in terms of predicting transplant-free survival. Patients were stratified according to tertiles of each marker and survival was determined by Kaplan-Meier analyses. All extracellular matrix remodeling markers showed significantly shorter transplant- 
free survival time for the higher tertile as compared to the middle and lower tertiles (PRO-C3: 1.83 [95\% Cl 1.36-2.30]; 3.35 [2.99-3.72]; 3.54 [3.19-3.88] years; $p<0.0001 ;$ PRO-C5: 2.20 [1.70-2.70]; 2.72 [2.51-3.19]; 3.74 [3.49-3.99] years; $p<0.0001 ;$ C3M: 2.31 [1.82-2.81]; 2.95 [2.51-3.39]; 3.45 [3.08-3.81] years; $p=0.0029$; C4M: 2.06 [1.56-2.56]; 3.07 [2.66-3.49]; 3.57 [3.25-3.90] years; $p<0.0001$ ) (Figure 2). Also patients in the higher tertile of the ELF Test had significantly shorter transplant-free survival time as compared to patients in the middle and lower tertiles (1.89 [1.41-2.37]; 3.20 [2.80-3.60]; 3.56 [3.22-3.89] years; $p<0.0001)$.

Similar to PRO-C3, PRO-C5, C3M, C4M and ELF Test, patients with higher tertile of the individual components of ELF Test had shorter transplant-free survival time, i.e. PIIINP (1.87 [1.39-2.35]; 3.20 [2.79$3.60]$; 3.65 [3.35-3.94] years; $p<0.0001)$, hyaluronic acid (1.88 [1.41-2.35]; 3.19 [2.79-3.59]; 3.55 [3.21-3.90] years; $p<0.0001)$, and TIMP1 (1.67 [1.19-2.14]; 3.21 [2.80-3.62]; 3.79 [3.60-3.98] years; $p<0.0001)$ (Supplementary Figure 2).

\section{Determination of independent prognostic markers in PSC by Cox regression analyses}

Univariate Cox proportional hazards regression analyses showed that all of the fibrosis markers were significantly associated with transplant-free survival $(p<0.05)$ (Table 4). Clinical characteristics (age, age at diagnosis, PSC duration), standard laboratory tests (bilirubin, AST, albumin and thrombocytes), the APRI score for fibrosis, the clinical PSC-specific Mayo risk score and the ELF Test were also associated with prognosis as previously described for this patient panel (Table 4). PRO-C3 was associated with the highest risk of reaching the primary endpoint of the extracellular matrix remodeling markers with an HR of 3.02 $(95 \% \mathrm{Cl}[1.96,4.67])$. Analyses in subsets excluding either patients with PSC with features of autoimmune hepatitis or patients with advanced disease as defined by Mayo risk score $>2$, showed similar significant associations with clinical outcome for the novel fibrosis markers (Supplementary Table 1 and 2). 
Using multivariable Cox regression analyses we investigated whether any of the markers were independently associated with transplant-free survival. In a first model (Supplementary Table 3), we included the four novel extracellular matrix remodeling markers, demonstrating that only the collagen formation markers, PRO-C3 and PRO-C5, were independently associated $(p<0.05)$ with clinical outcome. Multivariable analysis of a "Basic model" including all of the variables associated with clinical outcome in the univariate analysis, except the novel extracellular matrix markers and excluding redundant variables, resulted in a final model which included age at diagnosis, Mayo risk score and the ELF Test as independent predictors. Further multivariable analyses including the "Basic model" and each of the novel markers (in four separate analyses) showed that PRO-C5 and C4M were both independent predictors of clinical outcome, with final models including PRO-C5, ELF Test and age at diagnosis (HR 1.92, 1.69, and 1.04, respectively) and C4M, ELF Test and age at diagnosis (HR 1.98, 1.70, and 1.04, respectively), respectively, whereas PRO-C3 and C3M were not part of any final models (Supplementary Table 3). Because of the collinearity between PRO-C3 and ELF Test (and the biological overlap, both tests reflecting collagen III, either singularly or partly), we also performed an analysis of PRO-C3, and the "Basic model" with ELF Test removed (i.e., PRO-C3 and Mayo score, age at diagnosis, sex, Ln ALP), but PRO-C3 was still excluded from the final model. In a final step, we performed an analysis comprising the "Basic model" and all of the four novel markers, showing that only PRO-C5 (HR 1.92) out of the novel markers remained independently associated with clinical outcome, resulting in a final model comprising age at diagnosis, ELF test and Pro-C5 (Table 5).

Furthermore, we calculated the odds ratio for transplant-free survival in PSC comparing the lower tertile with the higher tertile of each marker. The ELF Test predicted transplant-free survival with an odds ratio of 11.3 (Figure 3). The two formation markers, PRO-C3 and PRO-C5, showed a slightly higher odds ratio as compared to the ELF Test (13.8 and 12.5, respectively), whereas the two degradation markers, C3M and 
C4M, showed a slightly lower odds ratio as compared to the ELF Test (6.1 and 8.7, respectively). Combining the two formation markers increased the odds ratio for predicting transplant-free survival to 47.3.

Finally, defining a secondary end-point as death or liver transplantation due to hepatic decompensation (endpoint reached by $n=25[18 \%]$ ), we found that the discriminatory ability of all four extracellular matrix remodeling biomarkers were reproduced confirming the strengths of Pro-C3 (Supplementary Table 4). ProC3 was associated with the highest risk of reaching the secondary endpoint ( $\mathrm{HR} 3.59,95 \% \mathrm{Cl}[1.89,6.81]$; Supplementary Table 5) for the univariate analyses, showing a HR higher than ELF test and Mayo score, respectively. In a multivariate analysis comprising the four novel extracellular matrix markers, Pro-C3 and C4M remained independently associated with clinical outcome; however, in the analysis encompassing the "Basic model" and all four novel extracellular matrix markers, none of the novel markers remained in the final model.

\section{DISCUSSION}

In this first study of four highly specific serological markers of extracellular matrix remodeling in PSC, reflecting the dynamic balance between fibrogenesis and fibrinolysis, we have shown that markers of extracellular matrix formation as well as degradation are elevated in PSC compared to ulcerative colitis, and associated with clinical outcome. Markers of fibrosis formation were stronger predictors of prognosis compared to degradation markers.

PRO-C3, a marker of collagen III and interstitial matrix formation, showed strong association with clinical outcome in Kaplan-Meier plot analysis and was the strongest prognostic predictor of the novel markers in univariate analysis. This is in line with findings in other etiologies of chronic liver disease such as chronic 
hepatitis $\mathrm{C}$ and confirms the potent predictive abilities of PRO-C3 $(14,18)$. Furthermore, our findings are in line with previous publications indicating that in PSC, serological and imaging markers of fibrogenesis such as the ELF Test, Fibroscan ${ }^{\circledR}$ and magnetic resonance elastography $(6,7,28)$ predict prognosis and are stronger predictors of clinical outcome compared with markers of inflammation (29). In the present study, AUROC analysis did not reveal significant differences between PRO-C3 and ELF Test in the discrimination between patients who did and did not reach an endpoint (primary or secondary endpoint, respectively). PRO-C3 was strongly correlated both to the ELF Test and to the clinical PSC-specific Mayo risk score (a widely used tool for risk stratification in PSC research). The complex interactions between these tightly correlated variables presumably contributed to the fact that PRO-C3 fell out of the final model in the comprehensive multivariate analysis. In the present patient panel, characterized by relatively short followup and high event-rate, the discriminatory ability of the Mayo score was excellent and the predictive power of Pro-C3 should be explored in a less advanced patient panel with fewer events and longer follow-up, situations where the Mayo score has shown inadequate discriminatory performance. PRO-C3 is a strong single marker of specific elements of the interstitial matrix formation and the value of combining PRO-C3 with other markers in order to define a novel marker panel should be further explored.

PRO-C5, a marker of interstitial matrix formation, was associated with the primary endpoint in this PSC patient panel and predicted prognosis in univariable analysis although with a slightly lower HR compared to PRO-C3 (HR 2.40 vs 3.02, respectively). In multivariable analyses PRO-C5 and C4M, but not PRO-C3 or C3M, predicted prognosis independently of Mayo risk score and ELF Test. PRO-C5 was the single marker out of the four novel markers to remain independently associated with clinical outcome in the final comprehensive multivariable analysis using the primary endpoint; however, these results regarding Pro-C5 were not reproduced in analyses using the secondary endpoint, and should be interpreted with caution. In contrast to PRO-C3, PRO-C5 was only weakly $(r<0.5)$ correlated to ELF Test and Mayo risk score, indicating 
that this marker reflects different aspects of the disease. Furthermore, in Kaplan-Meier plot analysis PROC5 seemed to segregate a particular low-risk group as opposed to PRO-C3 and the ELF Test, which rather seemed to delineate high-risk groups. Similarly, Mayo risk score is based on several variables reflecting advanced-stage liver disease. Distinguishing a low-risk group in less need of follow-up could be clinically useful. Type V collagen is a key determinant in collagen assembly of tissue specific matrices, including type I and III collagen (30), and can modify the stiffness of extracellular matrix, thereby affecting basic cellular functions (31). In the liver, type V collagen is found as part of extracellular matrix in the Space of Disse and portal tracts in line with type I, III, and IV collagen (32). In contrast to type I collagen, which only increase up to 1.6 fold in cirrhotic livers, type $V$ collagen increase with up to 4.6 fold. This indicates, that type $V$ collagen could be a more sensitive indicator of cirrhosis development $(33,34)$, which might explain the ability of PRO-C5 as prognostic biomarker related to hard end-points, such as death and liver transplantation. Finally, combination of PRO-C3 and PRO-C5 significantly increased the odds ratio to discriminate between patients with and without clinical outcome, demonstrating the value of combining markers to form biomarker panels.

Our results showed that markers of extracellular matrix degradation represented by C3M and C4M were also elevated in PSC patients compared to ulcerative colitis controls, reflecting increased extracellular matrix turnover. These degradation markers were also associated with transplant-free survival, in particular, C4M was associated with clinical outcome independently of both the Mayo risk score and ELF Test. Thickening of the biliary basement membrane is a hallmark of PSC, however the specific pathogenesis is uncertain (28). It is anticipated that epithelial cell damage and death result in disruption and destruction of the basement membrane (35). Following disruption of the basement membrane the underlying connective tissue, mainly composed on fibrillar collagens such as type I, III, and V collagen, becomes fibrotic driven by activated hepatic stellate cells and Kupffer cells synthesizing excessive amount of collagens and 
matrix metalloproteinases. Adjacent bile ducts are then surrounded by fibroblasts depositing thick bundles of fibrillar collagens ultimately leading to a thickening of the basement membrane giving rise to the characteristic onion skin appearance $(28,35)$. This hypothesis can to some extent be confirmed by the findings in our study. The initial injury is described by an increase in C4M levels and may partly explain the less prognostic potential of this marker, as it may reflect disease onset rather than disease progression. Conversely, the interstitial matrix formation markers PRO-C3 and PRO-C5, which have a large prognostic potential, reflect later disease stages and progression of fibrosis, which may lead to endpoints such as cirrhosis or death.

The results of our study confirm the pivotal role of fibrogenesis in PSC prognostication based on its important role in the pathogenesis of PSC. It is a well-recognized fact that progression to cirrhosis represents a worsening of prognosis, a fact also reflected by the PSC-specific Mayo risk score, presently the most commonly used prognostic score in PSC research and based on clinical and laboratory parameters reflecting cirrhosis and late-stage liver disease (bilirubin, albumin, INR, variceal bleeding). However, there is a major need in PSC research to develop biomarkers that delineate low-risk groups. The power of the novel fibrosis formation markers to delineate such low-risk groups should be further explored.

In contrast to previous studies, our data focus on the fact that fibrogenesis is a dynamic and complex process involving formation and deposition of a multitude of extracellular matrix constituents as well as degradation, in several compartments, and the relative importance of each of these simultaneously ongoing processes may vary between etiologies of hepatic fibrosis as well as over time (depending on disease activity) or between different stages of disease. If an injury is initiated by damage to the endothelial cells and the underlying basement membrane, then one could speculate, that remodeling of the basement membrane may reflect early fibrosis in PSC, whereas remodeling of the interstitial matrix may reflect later 
stages of fibrosis. This is not captured by imaging, which rather yields a static representation of the amount of fibrous tissue (i.e. stage) than an assessment of disease activity and progression rate. The ELF Test is a biomarker panel for generic liver fibrosis based on three direct components of fibrogenesis: hyaluronic acid, TIMP-1 and intact N-terminal PIIINP; however, does not discriminate between extracellular matrix formation and degradation. The PRO-C3 assay differs from the commonly used assays for PIIINP in that it is highly specific for type III procollagen synthesis (23). PRO-C3 targets the cleaved site of the N-terminal procollagen, thus allowing differentiation between formation and level of collagen III. This is in contrast to standard PIIINP assays which indiscriminately reflect type III collagen peptides of any kind. It is interesting to speculate that this may yield an increased sensitivity for changes in extracellular matrix remodeling for PRO-C3 over PIIINP assays and thus a potential improved reflection of disease activity as opposed to stage of liver fibrosis. This suggests a putative role of PRO-C3 as a surrogate marker in clinical trials which are currently hampered by the lack of approved, validated surrogate markers, however this should be further assessed in future studies.

Conceivably, the dissection between various aspects of fibrogenesis to elucidate which components or processes are the most important for PSC specifically may also enable the development of a PSC-specific prognostic testing tool with improved predictive ability over existing generic tests.

The lack of histological or radiological staging at the time of serum sampling and the retrospective study design represent limitations to our study. However, liver biopsy is not indicated in standard diagnosis or follow-up in PSC, and radiological staging is not well established; elastography was not available at the inclusion center in the time period investigated. Our data is limited by relatively short follow-up, prohibiting firm conclusions regarding low-risk groups. All patients in the present series were included at a tertiary referral center, potentially causing selection bias. Confirmation of our findings in an independent validation 
panel is warranted. The choice of all-cause death and liver transplantation as the primary combined endpoint may introduce elements of uncertainty based on variable indications for liver transplantation and non-liver related deaths; however, this is a commonly accepted end-point in the field and avoids uncertainties of missing data and varying interpretations inherent to other endpoints, and furthermore, analyses using a secondary endpoint comprising death and transplantation due to liver decompensation reproduced findings, in particular all of the findings regarding Pro-C3.

Employing serological biomarkers specifically targeting the extracellular matrix remodeling, we have demonstrated that markers of extracellular matrix formation are more important predictors of prognosis than markers of extracellular matrix degradation in PSC. We found that the extracellular matrix formation markers, particularly PRO-C3, were strong predictors of transplant-free survival in PSC. Further validation in independent PSC populations is warranted, and the fibrosis fingerprint of PSC should be further explored in order to identify the set of extracellular matrix markers that best capture the progression of fibrosis, and hence prognosis, in PSC, alone or in combination with clinically based scores such as the Mayo risk score or ALP.(36) Moreover, based on their reflection of the dynamic process of extracellular matrix remodeling, exploration of the potential role of these biomarkers as surrogate endpoints in clinical trials is warranted. 


\section{REFERENCES}

1. Karlsen $\mathrm{TH}$, Vesterhus $\mathrm{M}$, Boberg $\mathrm{KM}$. Review article: controversies in the management of primary biliary cirrhosis and primary sclerosing cholangitis. Aliment Pharmacol Ther. 2014 Feb;39(3):282301.

2. Montano-Loza AJ, Bhanji RA, Wasilenko S, Mason AL. Systematic review: recurrent autoimmune liver diseases after liver transplantation. Aliment Pharmacol Ther. 2017 Feb;45(4):485-500.

3. Boonstra K, Weersma RK, van Erpecum KJ, Rauws EA, Spanier BWM, Poen AC, et al. Populationbased epidemiology, malignancy risk, and outcome of primary sclerosing cholangitis. Hepatology. 2013;58:2045-2055.

4. Broomé U, Olsson R, Lööf L, Bodemar G, Hultcrantz R, Danielsson A, et al. Natural history and prognostic factors in 305 Swedish patients with primary sclerosing cholangitis. Gut. 1996;38:610-5.

5. de Vries EMG, Beuers U, Ponsioen CY. Biomarkers for disease progression of primary sclerosing cholangitis. Curr. Opin. Gastroenterol. 2015;31:239-246.

6. Vesterhus M, Hov JR, Holm A, Schrumpf E, Nygård S, Godang K, et al. Enhanced Liver Fibrosis Score Predicts Transplant-Free Survival in Primary Sclerosing Cholangitis. Hepatology. 2015;62:188-197.

7. de Vries EMG, Färkkilä M, Milkiewicz P, Hov JR, Eksteen B, Thorburn D, et al. Enhanced liver fibrosis test predicts transplant-free survival in primary sclerosing cholangitis, a multi-centre study. Liver Int. 2017;1-8.

8. Portmann B, Zen Y. Inflammatory disease of the bile ducts-cholangiopathies: Liver biopsy challenge and clinicopathological correlation. Histopathology. 2012;60:236-248.

9. Bosman FT, Stamenkovic I. Functional structure and composition of the extracellular matrix. J. Pathol. 2003;200:423-428. 
10. Karsdal M a, Nielsen MJ, Sand JM, Henriksen K, Genovese F, Bay-Jensen A-C, et al. Extracellular matrix remodeling: the common denominator in connective tissue diseases. Possibilities for evaluation and current understanding of the matrix as more than a passive architecture, but a key player in tissue failure. Assay Drug Dev. Technol. 2013;11:70-92.

11. Leeming DJ, Karsdal MA, Byrjalsen I, Bendtsen F, Trebicka J, Nielsen MJ, et al. Novel serological neoepitope markers of extracellular matrix proteins for the detection of portal hypertension. Aliment. Pharmacol. Ther. 2013;38:1086-1096.

12. Nielsen MJ, Kazankov K, Leeming DJ, Karsdal MA, Krag A, Barrera F, et al. Markers of collagen remodeling detect clinically significant fibrosis in chronic hepatitis C patients. PLoS One. 2015;10.

13. Nielsen MJ, Karsdal MA, Kazankov K, Grønbaek H, Krag A, Leeming DJ, et al. Fibrosis is not just fibrosis - basement membrane modelling and collagen metabolism differs between hepatitis B- and C-induced injury. Aliment. Pharmacol. Ther. 2016;44:1242-1252.

14. Nielsen MJ, Veidal SS, Karsdal MA, Ørsnes-Leeming DJ, Vainer B, Gardner SD, et al. Plasma Pro-C3 ( $\mathrm{N}$-terminal type III collagen propeptide) predicts fibrosis progression in patients with chronic hepatitis C. Liver Int. 2015;35:429-437.

15. Nielsen MJ, Lehmann J, Leeming DJ, Schierwagen R, Klein S, Jansen C, et al. Circulating Elastin Fragments Are Not Affected by Hepatic, Renal and Hemodynamic Changes, But Reflect Survival in Cirrhosis with TIPS. Dig. Dis. Sci. 2015;60:3456-3464.

16. Leeming DJ, Anadol E, Schierwagen R, Karsdal M a., Byrjalsen I, Nielsen MJ, et al. Combined antiretroviral therapy attenuates hepatic extracellular matrix remodeling in HIV patients assessed by novel protein fingerprint markers. Aids. 2014;28:2081-2090.

17. Karsdal MA, Henriksen K, Genovese F, Leeming DJ, Nielsen MJ, Riis BJ, et al. Serum endotrophin identifies optimal responders to PPARY agonists in type 2 diabetes. Diabetologia. 2016;1-10. 
18. Karsdal MA, Henriksen K, Nielsen MJ, Byrjalsen I, Leeming DJ, Gardner S, et al. Fibrogenesis assessed by serological type III collagen formation identifies patients with progressive liver fibrosis and responders to anti-fibrotic therapy. Am. J. Physiol. Gastrointest. Liver Physiol. 2016;ajpgi.00283.2016.

19. Chapman R, Fevery J, Kalloo A, Nagorney DM, Boberg KM, Shneider B, et al. Diagnosis and management of primary sclerosing cholangitis. Hepatology. 2010;51:660-678.

20. European Association for the Study of the Liver. EASL Clinical Practice Guidelines: Management of cholestatic liver diseases. J. Hepatol. 2009;51:237-267.

21. Solberg IC, Lygren I, Jahnsen J, Aadland E, Høie O, Cvancarova M, et al. Clinical course during the first 10 years of ulcerative colitis: results from a population-based inception cohort (IBSEN Study). Scand. J. Gastroenterol. 2009;44:431-440.

22. Wai CT, Greenson JK, Fontana RJ, et al. A simple noninvasive index can predict both significant fibrosis and cirrhosis in patients with chronic hepatitis C. Hepatology 2003. 38(2): p. 518-526

23. Nielsen MJ, Nedergaard AF, Sun S, Veidal SS, Larsen L, Zheng $Q$, et al. The neo-epitope specific PROC3 ELISA measures true formation of type III collagen associated with liver and muscle parameters. Am. J. Transl. Res. 2013;5:303-315.

24. Leeming DJ, Veidal SS, Karsdal MA, Nielsen MJ, Trebicka J, Busk T, et al. Pro-C5, a marker of true type $\mathrm{V}$ collagen formation and fibrillation, correlates with portal hypertension in patients with alcoholic cirrhosis. Scand. J. Gastroenterol. 2015;50:584-592.

25. Veidal SS, Vassiliadis E, Barascuk N, Zhang C, Segovia-Silvestre T, Klickstein L, et al. Matrix metalloproteinase-9-mediated type III collagen degradation as a novel serological biochemical marker for liver fibrogenesis. Liver Int. 2010;30:1293-1304.

26. Veidal SS, Karsdal MA, Nawrocki A, Larsen MR, Dai Y, Zheng Q, et al. Assessment of proteolytic 
degradation of the basement membrane: a fragment of type IV collagen as a biochemical marker for liver fibrosis. Fibrogenesis Tissue Repair. 2011;4:22.

27. Delong ER, Carolina N. Comparing the Areas under Two or More Correlated Receiver Operating Characteristic Curves : A Nonparametric Approach Author ( s ): Elizabeth R . DeLong, David M . DeLong and Daniel L . Clarke-Pearson Published by : International Biometric Society Stable . Biometrics. 2016;44:837-845.

28. Eaton JE, Talwalkar JA, Lazaridis KN, Gores GJ, Lindor KD. Pathogenesis of primary sclerosing cholangitis and advances in diagnosis and management. Gastroenterology. 2013;145:521-536.

29. Vesterhus M, Holm A, Hov JR, Nygård S, Schrumpf E, Melum E, et al. Novel serum and bile protein markers predict primary sclerosing cholangitis disease severity and prognosis. J. Hepatol. 2017;66:1214-1222.

30. Birk DE, Fitch JM, Babiarz JP, Doane KJ, Linsenmayer TF. Collagen fibrillogenesis in vitro: interaction of types I and V collagen regulates fibril diameter. J. Cell Sci. 1990;95 ( Pt 4):649-657.

31. Breuls RGM, Klumpers DD, Everts V, Smit TH. Collagen type V modulates fibroblast behavior dependent on substrate stiffness. Biochem. Biophys. Res. Commun. 2009;380:425-429.

32. Schuppan D, Becket J, Boehm H, Hahn EG. a n d T â€ $€^{\mathrm{TM}}$ e Immunofluorescent localization of the interstitial connective tissue of human oral mucosa , artery and liver. 1986;535-543.

33. Mak KM, Png CYM, Lee DJ. Type V Collagen in Health, Disease, and Fibrosis. Anat. Rec. 2016;299:613-629.

34. Takai KK, Hattori S, Irie S. Type V collagen distribution in liver is reconstructed in coculture system of hepatocytes and stellate cells; the possible functions of type $V$ collagen in liver under normal and pathological conditions. Cell Struct. Funct. 2001;26:289-302. 
35. Colling R, Verrill C, Fryer E, Kartsonaki C, Wang LM, Chapman R, et al. Bile duct basement membrane thickening in primary sclerosing cholangitis. Histopathology. 2016;68:819-824.

36. Rupp C, Rössler A, Halibasic E, Sauer P, Weiss KH, Friedrich K, Wannhoff A, Stiehl A, Stremmel W, Trauner M, Gotthardt DN. Reduction in alkaline phosphatase is associated with longer survival in primary sclerosing cholangitis, independent of dominant stenosis.Aliment Pharmacol Ther. 2014 Dec;40(11-12):1292-301. 
TABLES

Table 1: Demographic characteristics and biochemical parameters in patients with PSC and UC.

\begin{tabular}{|c|c|c|}
\hline & PSC & UC \\
\hline $\mathbf{N}$ & 138 & 52 \\
\hline Males, n (\%) & $107(77.5 \%)$ & $\mathrm{NI}$ \\
\hline Age, years, median (range) & $40(16-72)$ & $48(30-82)$ \\
\hline Age at diagnosis, median (range) & $34(14-72)$ & NA \\
\hline PSC duration, years, median (range) & $1.6(-0.6-29.0)$ & NA \\
\hline Features of AlH & $15(10.9 \%)$ & NA \\
\hline IBD ever, $\mathrm{n}(\%)$ & $102(74.4 \%)$ & $52(100 \%)$ \\
\hline Ulcerative colitis, $\mathbf{n}$ (\% of all) & $81(59.1 \%)$ & $52(100 \%)$ \\
\hline Liver transplantation, $\mathrm{n}(\%)$ & $33(23.9 \%)$ & NA \\
\hline Death, $n(\%)$ & $16(11.6 \%)$ & NA \\
\hline $\begin{array}{l}\text { Time to follow-up, months, median } \\
\text { (range) }\end{array}$ & $25.9(0.2-51.5)$ & NA \\
\hline Mayo risk score, median (range) & $0.12(-2.37-4.13)$ & NA \\
\hline ALP, U/L, median (range) & $224(51-1459)$ & $\mathrm{NI}$ \\
\hline ALT, U/L, median (range) & $85(14-885)$ & $\mathrm{NI}$ \\
\hline AST, U/L, median (range) & $68(16-1219)$ & $\mathrm{NI}$ \\
\hline Albumin, g/L, median (range) & $41(23-50)$ & $\mathrm{NI}$ \\
\hline $\begin{array}{l}\text { Total bilirubin, } \mu \mathrm{mol} / \mathrm{L}, \text { median } \\
\text { (range) }\end{array}$ & $20(3-532)$ & $\mathrm{NI}$ \\
\hline INR, median (range) & $1.0(0.8-1.8)$ & $\mathrm{NI}$ \\
\hline Platelets, $10^{9} / \mathrm{L}$, median (range) & $284(22-903)$ & $\mathrm{NI}$ \\
\hline
\end{tabular}


ALP, alkaline phosphatase; ALT, alanine aminotransferase; AST, aspartate aminotransferase; IBD, inflammatory bowel disease; INR, international normalized ratio; NA, not available; NI, not investigated. 
Table 2: AUROC analyses of ELF Test and extracellular matrix remodeling markers for detecting patients with end-points.

\begin{tabular}{|c|c|c|c|c|c|c|c|c|c|c|c|}
\hline & Prev & Marker & AUC & $\begin{array}{l}\text { Cut- } \\
\text { off }\end{array}$ & Sens & Spec & PPV & NPV & $+\mathrm{LR}$ & LR- & p-value \\
\hline & $34 \%$ & ELF & 0.81 & $>10.6$ & 70 & 79 & 64 & 84 & 3.4 & 0.4 & $<0.0001$ \\
\hline Patients with vs. & & Test & & & & & & & & & \\
\hline without end- & & PRO-C3 & 0.78 & $>35.1$ & 74 & 81 & 67 & 86 & 4.0 & 0.3 & $<0.0001$ \\
\hline \multirow[t]{4}{*}{ point } & & PRO-C5 & 0.74 & $>626.9$ & 87 & 57 & 51 & 90 & 2.0 & 0.2 & $<0.0001$ \\
\hline & & C3M & 0.75 & $>36.7$ & 74 & 74 & 59 & 85 & 2.8 & 0.4 & $<0.0001$ \\
\hline & & C4M & 0.76 & $>120.6$ & 72 & 74 & 59 & 84 & 2.7 & 0.4 & $<0.0001$ \\
\hline & & APRI & 0.70 & $>0.5$ & 74 & 61 & 50 & 82 & 1.9 & 0.4 & $<0.001$ \\
\hline \multicolumn{12}{|c|}{ A combined endpoint was defined as all-cause death or liver transplantation. Prev, prevalence i.e. patients } \\
\hline \multicolumn{12}{|c|}{ with end-point; AUC, Area under curve, Sens, sensitivity, spec, specificity, PPV, positive predictive value; } \\
\hline \multicolumn{12}{|c|}{$N P V$, negative predictive value; $+L R$, positive likelihood ratio; $-L R$, negative likelihood ratio; APRI, aspartate } \\
\hline
\end{tabular}


Table 3: Median survival time for PSC patients in "high risk" group and "low risk" group. PSC patients were divided in the two groups based on the cut-off level determined by the Youden index in the AUROC analyses in Table 2.

\begin{tabular}{|l|l|l|l|l|l|l|l|}
\hline \multirow{2}{*}{} & \multicolumn{3}{|c|}{ Low risk group } & \multicolumn{3}{c|}{ High risk group } & \multirow{2}{*}{ P-value } \\
\cline { 2 - 8 } & Median years & \multicolumn{1}{|c|}{ IQR } & $\mathbf{n}$ & Median years & IQR & $\mathbf{n}$ & \\
\hline ELF Test & 2.72 & $1.30-3.61$ & 85 & 1.42 & $0.32-2.41$ & 53 & $<0.001$ \\
\hline PRO-C3 & 2.56 & $1.38-3.28$ & 85 & 1.25 & $0.30-3.01$ & 53 & 0.001 \\
\hline PRO-C5 & 2.77 & $1.70-3.32$ & 58 & 1.55 & $0.40-2.94$ & 80 & $<0.001$ \\
\hline C3M & 2.32 & $1.15-3.22$ & 78 & 1.69 & $0.39-3.05$ & 60 & 0.059 \\
\hline C4M & 2.58 & $1.15-3.55$ & 79 & 1.54 & $0.38-2.83$ & 59 & 0.002 \\
\hline
\end{tabular}

AUROC, area under the receiver operating characteristic curve; IQR, interquartile range; ELF Test, Enhanced Liver Fibrosis Test. 
Table 4. Univariate Cox regression analyses of factors associated with transplant-free survival in PSC

patients ( $n=138$ ). Cox proportional-hazards regression rates were calculated for tertiles of each of the novel extracellular matrix markers and ELF Test. PRO-C3 (collagen III formation marker) performed best out of the novel markers. Bilirubin, ALP, AST, ALT, and platelet count were transformed by the natural logarithm prior to regression analyses due to a right-skewed distribution.

\begin{tabular}{|c|c|c|c|c|c|}
\hline & HR & $95 \% \mathrm{Cl}$ & $P$ value & Wald & $\mathbf{N}$ \\
\hline Age & 1.05 & $1.02,1.07$ & $<0.001$ & & 138 \\
\hline Sex & 0.62 & $0.33,1.19$ & 0.151 & & 138 \\
\hline Age at diagnosis & 1.04 & $1.02,1.06$ & $<0.001$ & & 138 \\
\hline PSC duration & 1.03 & $0.99,1.07$ & 0.211 & & 138 \\
\hline IBD status ever & 1.57 & $0.85,2.89$ & 0.152 & & 137 \\
\hline Bilirubin & 2.24 & $1.73,2.88$ & $<0.001$ & & 130 \\
\hline Albumin & 0.86 & $0.82,0.91$ & $<0.001$ & & 129 \\
\hline ALP & 2.35 & $1.57,3.53$ & $<0.001$ & & 130 \\
\hline AST & 1.73 & $1.28,2.35$ & $<0.001$ & & 130 \\
\hline ALT & 1.17 & $0.84,1.63$ & 0.361 & & 130 \\
\hline Platelet count & 0.66 & $0.43,1.01$ & 0.053 & & 129 \\
\hline INR & 17.98 & $5.01,64.52$ & $<0.001$ & & 113 \\
\hline Variceal bleeding & 2.59 & $0.80,8.35$ & 0.112 & & 138 \\
\hline APRI score & 2.04 & $1.39,3.01$ & $<0.001$ & 13.1 & 129 \\
\hline
\end{tabular}




\begin{tabular}{lccccc} 
Mayo risk score & 2.06 & $1.67,2.54$ & $<0.001$ & 46.1 & 129 \\
ELF ${ }^{\circledR}$ Test & 2.90 & $1.90,4.41$ & $<0.001$ & 24.3 & 138 \\
& & & & & \\
PRO-C3 & 3.02 & $1.96,4.67$ & $<0.0001$ & 24.6 & 138 \\
PRO-C5 & 2.40 & $1.6,3.56$ & $<0.0001$ & 19.0 & 138 \\
& & & & & \\
C3M & 1.91 & $1.30,2.81$ & 0.001 & 10.8 & 138 \\
C4M & & & & & \\
& 2.44 & $1.63,3.65$ & $<0.0001$ & 18.8 & 138 \\
\hline
\end{tabular}

$\overline{A L P}$, alkaline phosphatase; ALT, alanine aminotransferase; AST, aspartate aminotransferase; APRI, AST to platelet index; IBD, inflammatory bowel disease; INR, international normalized ratio; $\mathrm{HR}$, hazard ratio; $\mathrm{Cl}$, confidence interval. 
Table 5: Multivariate Cox regression analyses to identify independent predictors of transplant-free survival in PSC patients. Cox proportional-hazards regression calculated for tertiles of each marker using forward elimination.

\begin{tabular}{|c|c|c|c|c|c|}
\hline \multicolumn{3}{|l|}{ Multivariate } & \multicolumn{3}{|c|}{ Wald } \\
\hline Cox* & HR & $95 \% \mathrm{Cl}$ & $P$ value & & $\mathbf{N}$ \\
\hline Age at diagnosis & 1.04 & $1.02,1.06$ & 0.001 & 11.3 & 128 \\
\hline Sex & - & - & - & - & \\
\hline Ln ALP & - & - & - & - & \\
\hline Mayo risk score & - & - & - & - & \\
\hline ELF Test & 1.69 & $1.39,2.06$ & $<0.001$ & 26.9 & \\
\hline PRO-C5 tertiles & 1.92 & $1.28,2.88$ & 0.002 & 10.0 & \\
\hline PRO-C3 tertiles & - & - & - & - & \\
\hline C3M & - & - & - & - & \\
\hline C4M & - & - & - & - & \\
\hline
\end{tabular}

ALP, alkaline phosphatase; $\mathrm{HR}$, hazard ratio; $\mathrm{Cl}$, confidence interval. 


\section{FIGURE LEGENDS}

\section{Figure 1:}

Serum levels of extracellular matrix remodeling is elevated in PSC patients compared to UC patients. A) Serum levels of type III collagen formation, PRO-C3; B) Serum levels of type V collagen formation, PRO-C5; C) Serum levels of type III collagen degradation, C3M; D) Serum levels of type IV collagen degradation, C4M, and D) Serum levels of the ELF Score. Data are shown as Tukey's boxplot. Outliers above or below 1.5 IQR have been included in analyses, but are left blank in the figures for better visualization of the medians. Asterisks indicate significant differences as indicated by bars: ${ }^{* *} \mathrm{p}<0.001$.

\section{Figure 2}

Prediction of transplant free survival by the four extracellular matrix remodeling markers and the ELF Test. The figure shows Kaplan-Meier curves of time of transplantation or death for PSC patients stratified into tertiles of A) PRO-C3, B) PRO-C5, C) C3M, D) C4M, and E) ELF Test.

\section{Figure 3}

Odds ratios (OR) for predicting transplant free survival in PSC patients. The figure shows OR with $95 \% \mathrm{Cl}$ for predicting transplant free survival in PSC patients in the highest baseline tertile serum level compared to patients with lowest baseline tertile serum level of the ELF Test, PRO-C3, PRO-C5, C3M, C4M, and a combination of PRO-C3 and PRO-C5. The dotted line represents the OR of ELF Test for comparison. Asterisks indicate significant differences as: ${ }^{* * *} p<0.001$. 
FIGURES

Figure 1
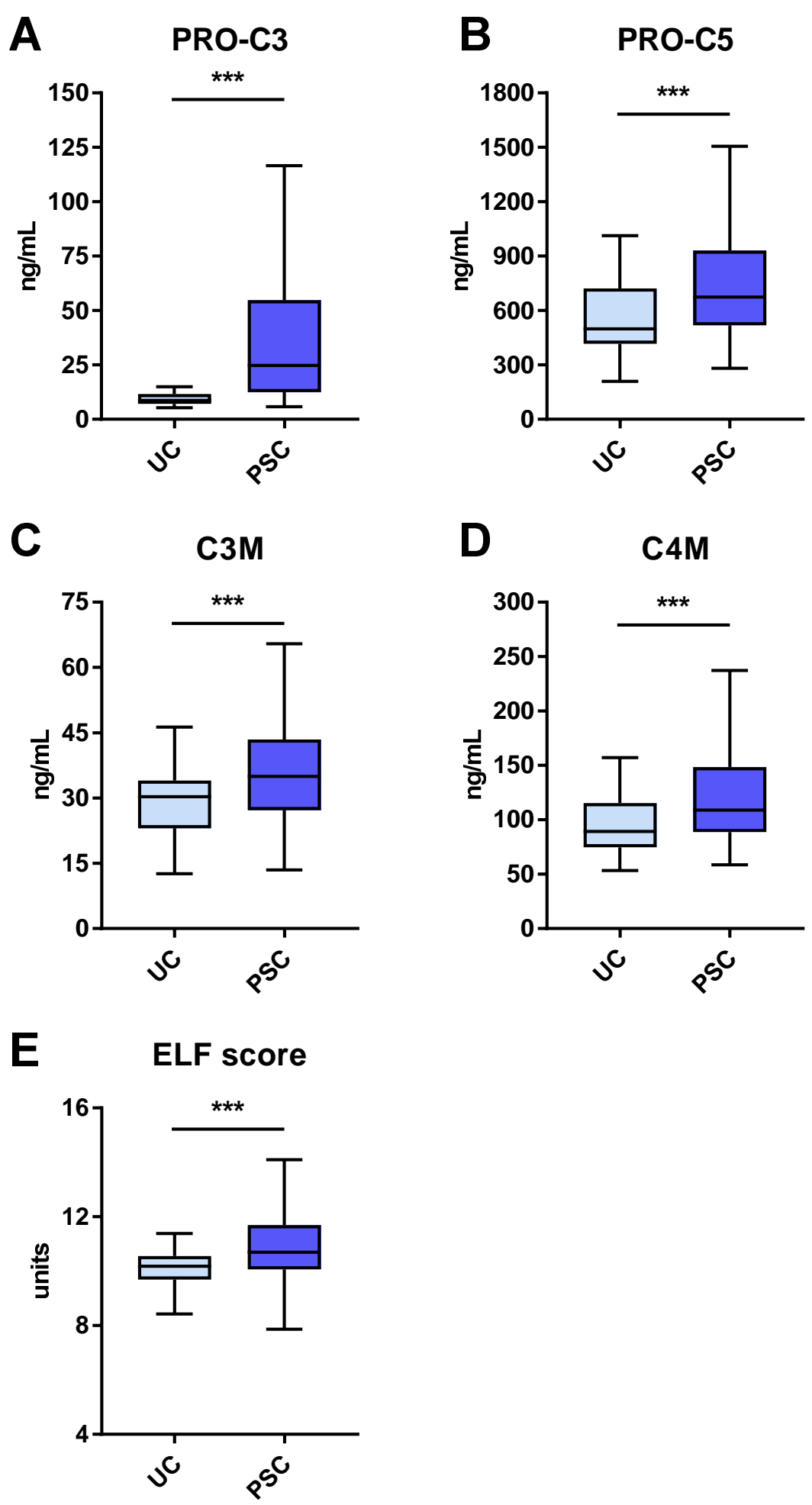
Figure 2

A

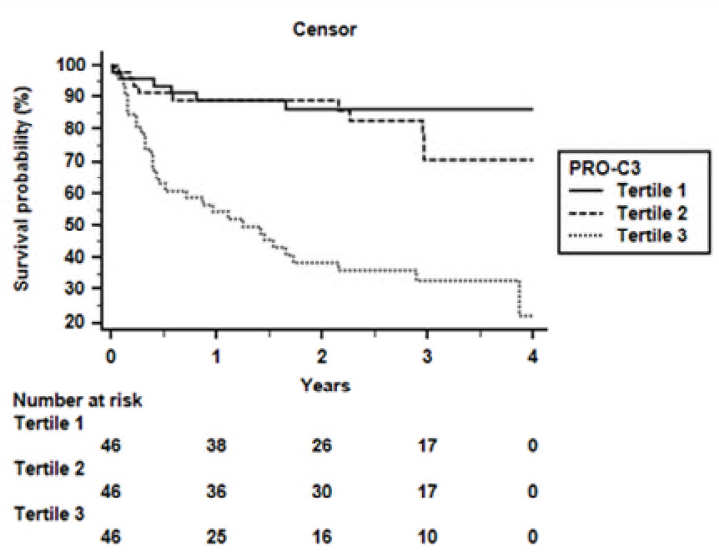

C

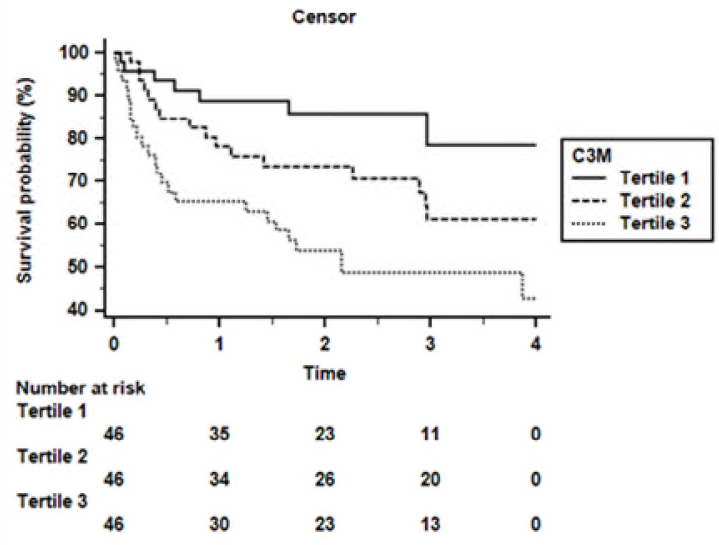

E

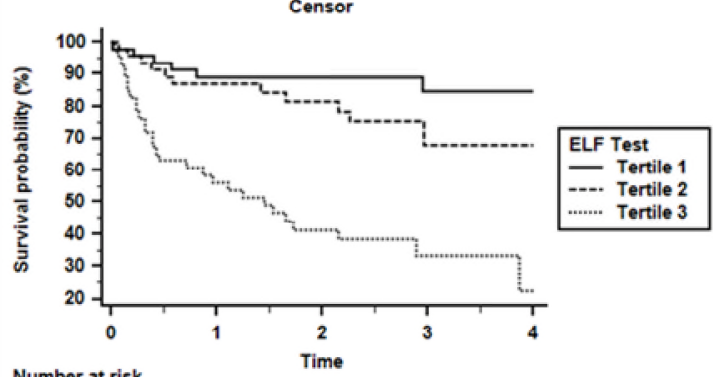

Number at risk

Tertile 1

Tertile 2 $46 \quad 38 \quad 30 \quad 20 \quad 0$

$\begin{array}{llllll}\text { Tertile 2 } & & & & & \\ & 46 & 37 & 27 & 18 & 0\end{array}$

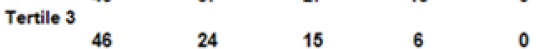

B

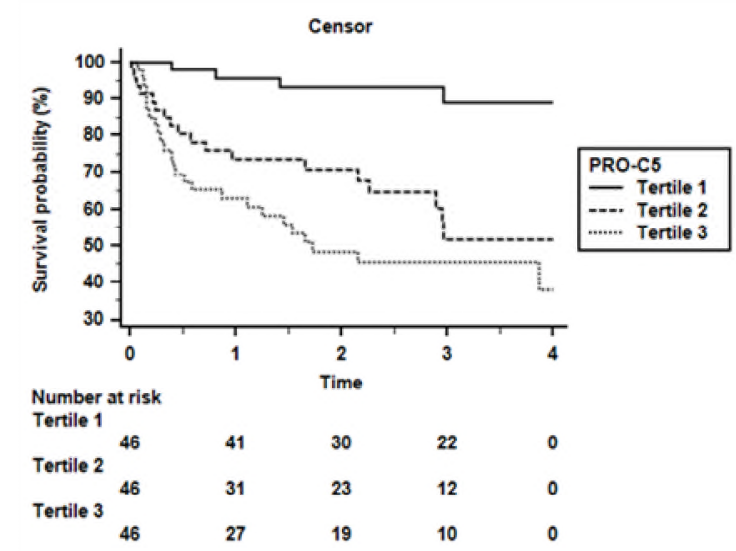

D

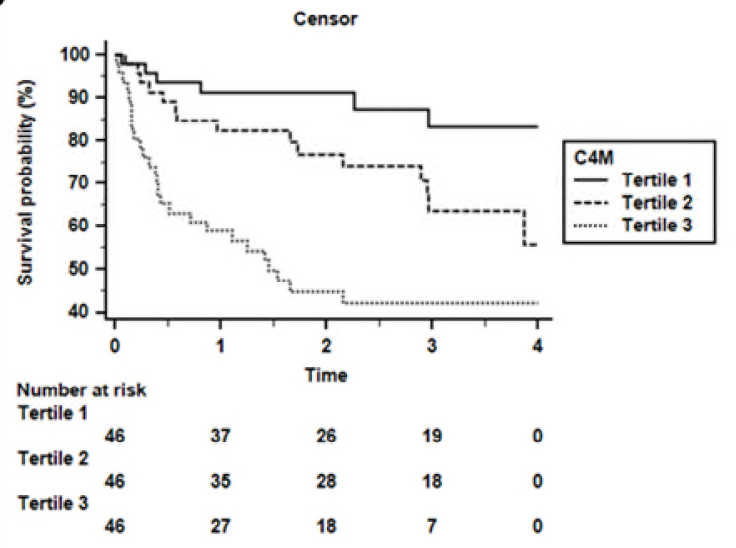


Figure 3

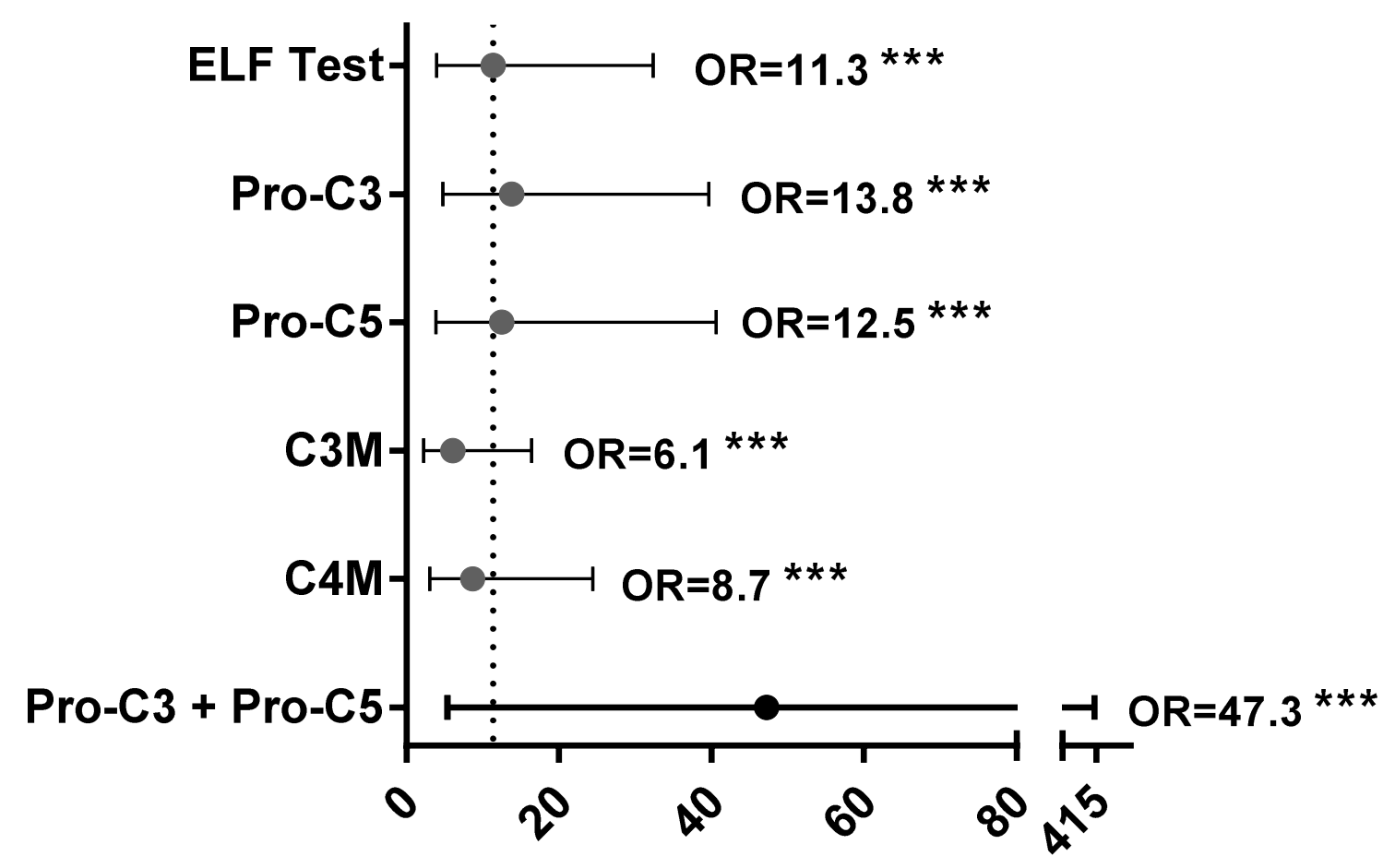

